\title{
ANALYSIS OF FLASH PULSE THERMOGRAPHIC INSPECTION PARAMETERS FOR QUANTITATIVE MEASUREMENT
}

\author{
${ }^{1}$ Michal ŠVANTNER, ${ }^{2}$ Lukáš MUZIKA, ${ }^{3}$ Alexey MOSKOVCHENKO \\ ${ }^{1}$ University of West Bohemia, Plzeň, Czech Republic, EU, msvantne@ntc.zcu.cz \\ 2 University of West Bohemia, Plzeň, Czech Republic, EU, muzika@ntc.zcu.cz \\ ${ }^{3}$ University of West Bohemia, Plzeň, Czech Republic, EU, moskovchenko.alexey@gmail.com
}

https://doi.org/10.37904/metal.2021.4184

\begin{abstract}
Flash-pulse thermography is a method for the detection of discontinuities or inhomogeneities in materials at their surface. It is based on excitation of inspected samples by a short pulse and analysis of its thermal response. It is basically an indicative method, however, quantitative procedures for an evaluation of defects detectability, defects depths or a thickness of coatings are also developed. The quantitative evaluation has, in general, higher demands on the accuracy of a measurement procedure. This contribution is focused on the analysis of parameters of recording of thermographic data. The influence of synchronization of a sample thermal response recording with an excitation source is analyzed. Differences between recording using a bolometric thermographic camera and a cooled detector based thermographic camera are demonstrated on flat-bottom hole samples. The results show that a high-level synchronization is crucial for the quantitative evaluation of flash-pulse thermography. It is also shown that the cooled quantum detector based thermographic cameras have better temperature response in the case of cooling process measurement and can produce higher sensitivity and lower noise records. Thus, it should be used for any quantitative flash-pulse thermography measurement, even if a bolometric detector type camera would satisfied framerates requirements.
\end{abstract}

Keywords: Active thermography, thermographic testing, flash-pulse thermography, infrared non-destructive testing

\section{INTRODUCTION}

Thermographic testing is an active thermography method, which is used for a near-surface material inspection. It is based on a thermal excitation of a measured object, which induces a thermal process. The process is influenced by defects or other inhomogeneities in the object if these are present. It can be reflected by a thermal response on an object surface. The defects can be indicated by an analysis of the thermal response by a suitable active thermography method. The methods and principles of the active thermography testing are more in detail described for example in Maldague [1], advances, historical progress and state-of-the-art are in Meola [2] or Vavilov [3].

Active thermography - infrared non-destructive testing (IRNDT) represents a very variable and flexible group of methods. Different excitation sources (flash lamps, halogen lamps, ultrasound etc.), excitation methods (pulse, periodical, step etc.) and evaluation procedures (temperature differences evaluation, principal components thermography, TSR - thermographic signal reconstruction, pulse-phase, lock-in etc.) can be used based on applications and other requirements. An overview of thermographic temperature measurements and IRNDT can be found, e.g., in [4] and [5]. The most common methods of pulsed thermography, which this contribution is focused on, are introduced in [6] or [7]. 
IRNDT methods are basically contrast-based methods. Their outputs are mostly temperature differences or differences of some temperature transformed quantity. The differences make regions with a different contrast, which can indicate possible defects or inhomogeneities in tested objects. However, the newest trends in active thermography are aimed at quantification of the results. This quantification can be focused on both quantitative evaluation of indications visibility (e.g., using contrast to noise ratio [8]) or additional quantification inspection results (e.g., defects depth estimation [9] or coating thickness inspection [10]). Limits of measurement equipment, parameters of the inspection and thermal response measurement accuracy can play a much more important role in such kind of evaluation than in the case of a simple contrast-based inspection with categoricalbinary outputs (defective/non-defective).

A used infrared camera is one of the variable parameters in flash-pulse IRNDT. For some applications, mostly in the case of high-diffusivity materials, it is necessary to measure a very short time range after the pulse with a high frame rate. Cooled high-speed IR cameras can be only used in this case. However, lower framerates than 50 or $25 \mathrm{~Hz}$ can be sufficient for low-diffusivity materials. In this case, the cooled (quantum detector based) IR camera could be replaced by a standard bolometric-type detector IR camera. These cameras are typically significantly cheaper. However, even though their framerate could be sufficient, their output signal is often noisier and mostly cannot be synchronized so precisely as the cooled high-speed IR cameras. Thus, this study is focused on a comparison of bolometric and high-speed IR cameras for a flash-pulse thermography sub-surface defects inspection of a steel part. The usability of cheaper bolometric IR cameras for these measurements is analyzed.

\section{EXPERIMENTS DESCRIPTION}

The flash pulse thermography inspection experiments were made on an AISI 446 steel specimen with flat bottom holes artificial defects. The specimen's dimensions were $200 \times 150 \mathrm{~mm}$, its thickness was $5.15 \mathrm{~mm}$. There were 22 flat bottom holes of different diameter and depth made from the bottom side of the specimen. The inspections were made from the top side of the specimen and the holes so simulated defects of the diameter and depth in ranges $0.8-9.9 \mathrm{~mm}$ and $0.15-4.15 \mathrm{~mm}$, respectively.

The experimental setup consisted of a flash lamp (Hensel EH Pro 6000), which excited the specimen from its top side with a flash pulse of the power $6 \mathrm{~kJ}$ and a length of about $6 \mathrm{~ms}$. Response of the specimen was measured by a bolometric detectors type camera Optris PI 640 and by a quantum detector type camera FLIR A6751. Regarding datasheets information the temperature measurement accuracy of both cameras was $\pm 2{ }^{\circ} \mathrm{C}$. However, NETD (Noise Equivalent Temperature Difference) of the Optris and FLIR cameras was $75 \mathrm{mK}$ and $25 \mathrm{mK}$, respectively. The flash lamp was perpendicular to the tested surface in the distance of 500 $\mathrm{mm}$ and the camera was at an angle of about $30^{\circ}$ in the distance of $550 \mathrm{~mm}$. Acquisition frequency (framerate) and length were $50 \mathrm{~Hz}$ and 1000 frames, respectively, for both cameras. Optris camera was not precisely synchronized, and the highest temperature of a record was taken as a start point. The FLIR A6751 camera was used without synchronization and/or synchronized with the flash pulse [10] with precision of about $2 \mu s$ and the results were compared.

The results of the inspections were processed by standard ways for thermographic testing - temperature, temperature contrast (differences) and processing by TDTS $1^{\text {st }}$ derivatives [11]. Those methods were compared for individual IR cameras.

\section{RESULTS AND DISCUSSION}

The raw results of the inspections are time-sequences of thermograms and/or defectograms. The thermograms and $1^{\text {st }}$ time derivative of thermographic signal (TDTS) maps obtained by the FLIR and the Optris cameras after selecting frames with the best contrast of detected indications are shown in Figure 1. Six or seven indications ( $5 / 6$ indications at the top, 1 indication at the bottom of the specimen, Figure $1 \mathrm{~A}$ and $\mathrm{C}$ ) can 
be seen on the raw thermogram images recorded by both FLIR and Optris cameras. It is also evident by a visual inspection, that a noise level is much higher at the Optris image than at the FLIR image. That is apparently the main reason that additional indications appear after $1^{\text {st }}$ TDTS processing of the FLIR record (6/7 indications at the top, $4 / 5$ indication at the center and $3 / 4$ indication at the bottom of the specimen, Figure $1 \mathrm{~B}$ ), but no additional indications can be observed after $1^{\text {st }}$ TDTS processing at the Optris image (Figure $1 \mathrm{D}$ ) although the indications are more contrast after processing than on the raw thermogram.

A) FLIR A6750, raw image

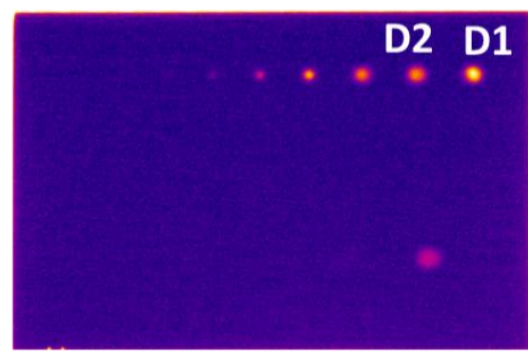

B) FLIR A6750, $1^{\text {st }}$ TDTS

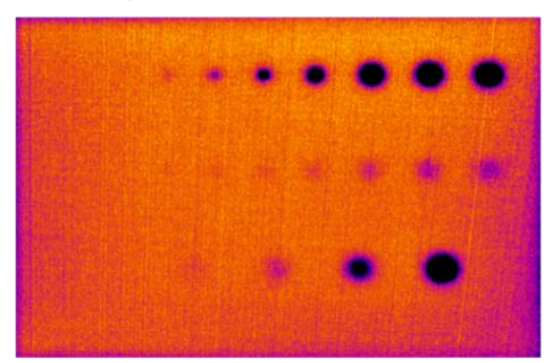

C) Optris, raw image

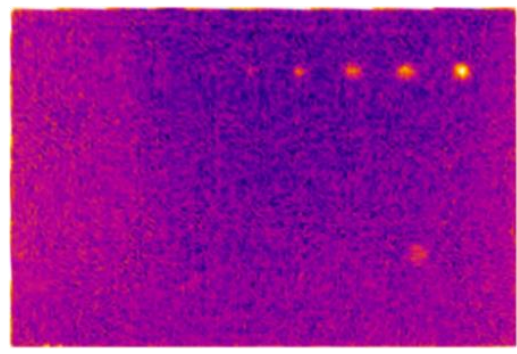

D) Optris, $1^{\text {st }}$ TDTS

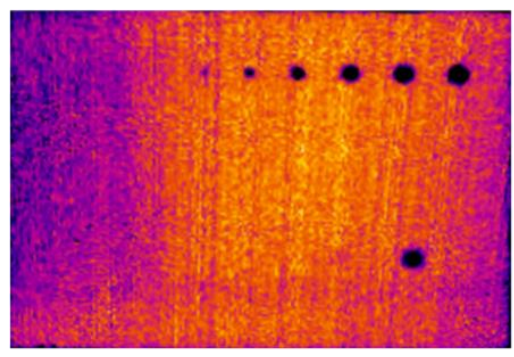

Figure 1 Thermograms and TDTS $1^{\text {st }}$ derivative defectograms of the flash pulse inspections of the steel specimen made using FLIR A6750 and Optris PI640 cameras.

The observation relating to the higher noise can be also confirmed by temperature-time profiles at individual points shown in Figure 2.

A )Temperature evolution

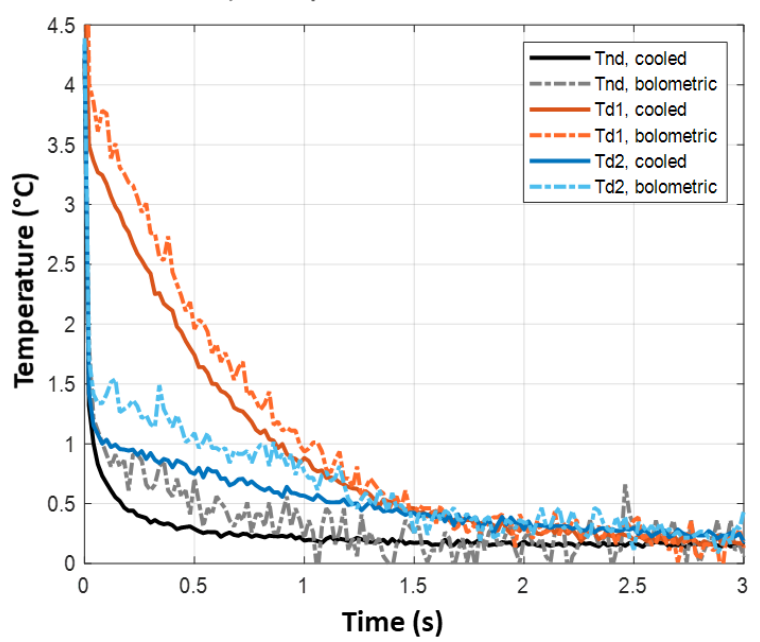

B) Temperature evolution Log scale

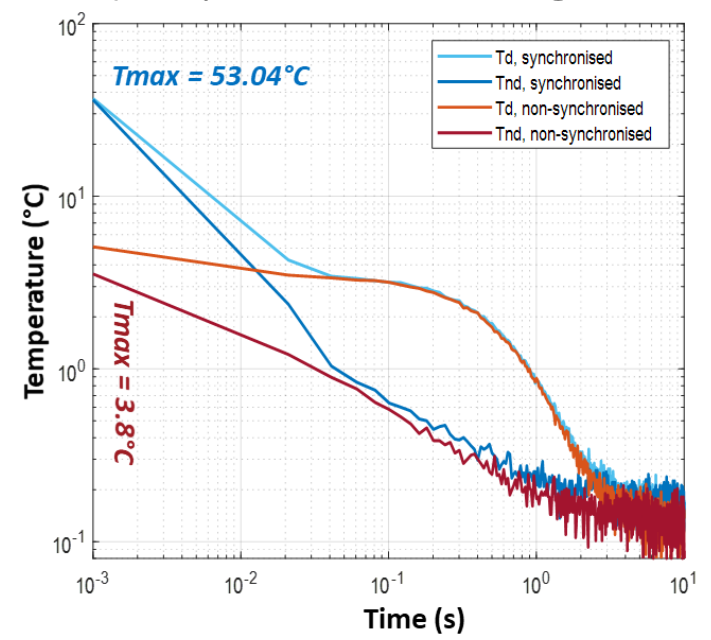

Figure 2 Time-temperature profile at a defect-free region and the positions of defects D1 and D2 measured by FLIR (cooled) and Optris (bolometric) cameras (A), time-temperature profile recorded by FLIR for a synchronized and non-synchronized mode at a defect-free region and a position of a defect (B). 
The thermograms/defectograms based observations relating the higher noise at Optris IR camera output is evident on temperature-time profiles for both defect-free and defects region (Figure 2 A). In addition, a delay/shift in the temperature evolution can be observed for the data measured by the Optris camera. This can be caused by a principle of micro-bolometric detectors used by the Optris camera, which are resistive radiantheat detectors and can have a longer response time compared to optical quantum-based detectors used by the cooled FLIR camera.

A comparison of temperature responses on defect-free and defect regions acquired by the FLIR camera in synchronized and non-synchronized modes are shown in Figure $2 \mathbf{B}$. The synchronization allows recording the very first frame during the heat pulse and gives precise information about time counts after the flash pulse. While unsynchronized sequence contains the flash pulse between the recorded frames and does not reflect the maximum initial temperature and exact time steps after the flash pulse. It can be observed in timetemperature progress in log-log plot in Figure 2 B.

\section{CONCLUSION}

The results of the performed analysis showed that both cooled quantum-detector based IR camera (FLIR A6751) and micro-bolometer type IR camera (Optris PI 640) produced similar raw thermographic records, where the same or a similar number of indications of sub-surface defects (6/7 of 22 total defects indicated) could be identified. However, it was shown that the cooled IR camera has a higher sensitivity and noticeably lower noise level that influenced the results of the subsequent processing by the TDTS procedure. This thermographic processing caused a contrast enhancement of the previously found indications for both cameras. It also revealed the next indications (together approximately 15 of 22 total defects indicated) using the FLIR camera records while the evaluation of the Optris IR camera record did not bring quantitative enhancement of the results.

The temperature delay/shift can be observed on the time-temperature data provided by the Optris IR camera. Another disadvantage of these IR cameras (bolometric-type detector cameras in general) is that they cannot be synchronized precisely enough with a flash-pulse. The presented comparison between synchronized and non-synchronized records performed for the FLIR camera showed that it can add additional errors and uncertainty to the results. These drawbacks together with higher noise can significantly - negatively influence the quantitative evaluation of results, for example, a set of an exact position of some specific points on timetemperature transformed curve, which is necessary for defect depth [9] or coating thickness [12] determination. Thus, it can be concluded that non-cooled bolometric-type IR cameras can be used for flash-pulse qualitative inspection if high sensitivity is not required but should not be used for high-accuracy quantitative measurements.

\section{ACKNOWLEDGEMENTS}

This The work has been supported by project ERDF "LABIR-PAV / Pre-application research of infrared technologies" (No. CZ.02.1.01/0.0/0.0/18_069/0010018) and project SGS-2019-008

\section{REFERENCES}

[1] X.P. V. MALDAGUE. Theory and practice of infrared technology for nondestructive testing. Wiley. 2001.

[2] MEOLA C. Recent Advances in Non-Destructive Inspection. Nova Science Publishers. [online]. 2011. https://books.google.cz/books?id=dtTjRgAACAAJ.

[3] VAVILOV, V. Thermal NDT: historical milestones, state-of-the-art and trends, Quant. Infrared Thermogr. J. [online]. 2014, vol. 11, pp. 66-83. Available from: https://doi.org/10.1080/17686733.2014.897016. 
[4] USAMENTIAGA, R., VENEGAS, P., GUEREDIAGA, J., VEGA, L., MOLLEDA, J., BULNES, F.G. Infrared Thermography for Temperature Measurement and Non-Destructive Testing. Sensors. [online]. 2014, vol. 14, pp. 12305-12348. Available from: https://doi.org/10.3390/s140712305.

[5] IBARRA-CASTANEDO, C., TARPANI, J.R., MALDAGUE, X.P. V. Nondestructive testing with thermography. Eur. J. Phys. [online]. 2013, vol. 34, pp. S91-S109. Available from: https://doi.org/10.1088/0143-0807/34/6/S91.

[6] BALAGEAS, D.L. Defense and illustration of time-resolved pulsed thermography for NDE, QIRT J. [online]. 2012, vol. 9, pp.3-32. Available from: https://doi.org/10.1080/17686733.2012.676902.

[7] MALDAGUE, X., GALMICHE, F, ZIADI, A. Advances in pulsed phase thermography. Infrared Phys. Technol. [online]. 2002, vol. 43, pp. 175-181. Available from: https://doi.org/10.1016/S1350-4495(02)00138-X.

[8] ŠVANTNER, M., MUZIKA, L., CHMELÍK, T., SKÁLA, J. Quantitative evaluation of active thermography using contrast-to-noise ratio. Appl. Opt. [online]. 2018, vol. 57, pp. D49-D55. Available from:

https://doi.org/10.1364/AO.57.000D49.

[9] MOSKOVCHENKO, A.I., VAVILOV, V.P., CHULKOV, A.O. Comparing the efficiency of defect depth characterization algorithms in the inspection of CFRP by using one-sided pulsed thermal NDT. Infrared Phys. Technol. INFPHY [online]. 2020, vol. 103. Available from: https://doi.org/10.1016/j.infrared.2020.103289.

[10] ŠVANTNER, M., MUZIKA, L., HOUDKOVÁ, Š. Quantitative inspection of coatings thickness by flash-pulse thermography and time-power transformation evaluation. Appl. Opt. [online]. 2020, vol. 59, pp. E29-E35. Available from: https://doi.org/10.1364/AO.388440.

[11] MUZIKA, L., ŠVANTNER, M., MOSKAL, D. Experimental investigation of a time-power transformation method for flash-pulse thermographic testing. Appl. Opt. [online]. 2020, vol. 59, pp. E89-E96. Available from: https://doi.org/10.1364/AO.388437.

[12] MUZIKA, L., ŠVANTNER, M., HOUDKOVÁ, Š., ŠULCOVÁ, P. Application of flash-pulse thermography methods for quantitative thickness inspection of coatings made by different thermal spraying technologies. Surf. Coatings Technol. [online]. 2020, vol. 406, p. 126748. Available from: https://doi.org/10.1016/j.surfcoat.2020.126748. 